\title{
Chinese Herbal Medicine Qi Ju Di Huang Wan for the Treatment of Essential Hypertension: A Systematic Review of Randomized Controlled Trials
}

\author{
Jie Wang, ${ }^{1}$ Xingjiang Xiong, ${ }^{1}$ Guoyan Yang, ${ }^{2}$ Yuqing Zhang, ${ }^{3}$ Yongmei Liu, ${ }^{1}$ Yun Zhang, \\ Zhenpeng Zhang, ${ }^{1}$ Jun $\mathrm{Li}^{1}{ }^{1}$ and Xiaochen Yang' \\ ${ }^{1}$ Department of Cardiology, Guang'anmen Hospital, China Academy of Chinese Medical Sciences, Beijing 100053, China \\ ${ }^{2}$ Centre for Evidence-Based Chinese Medicine, Beijing University of Chinese Medicine, Beijing 100029, China \\ ${ }^{3}$ Department of Clinical Epidemiology and Biostatistics, McMaster University, ON, Canada L8S 4L8
}

Correspondence should be addressed to Xiaochen Yang; avill1988@126.com

Received 18 January 2013; Revised 25 April 2013; Accepted 12 May 2013

Academic Editor: Myeong Soo Lee

Copyright (c) 2013 Jie Wang et al. This is an open access article distributed under the Creative Commons Attribution License, which permits unrestricted use, distribution, and reproduction in any medium, provided the original work is properly cited.

\begin{abstract}
Background. Chinese herbs are potentially effective for hypertension. Qi Ju Di Huang Wan (QJDHW) is a commonly used Chinese herbal medicine as a monotherapy or in combination with other antihypertensive agents for the treatment of essential hypertension $(\mathrm{EH})$. However, there is no critically appraised evidence such as systematic reviews or meta-analyses on the effectiveness and safety of QJDHW for EH. Methods and Findings. CENTRAL, PubMed, CBM, CNKI, VIP, and online clinical trial registry websites were searched for published and unpublished randomized controlled trials (RCTs) of QJDHW for essential hypertension up to January 2013 with no language restrictions. A total of 10 randomized trials involving 1024 patients were included. Meta-analysis showed that QJDHW combined with antihypertensive drugs was more effective in lowering blood pressure and improving TCM syndrome for the treatment of essential hypertension than antihypertensive drugs used alone. No trials reported severe adverse events related to QJDHW. Conclusions. Our review suggests that QJDHW combined with antihypertensive drugs might be an effective treatment for lowering blood pressure and improving symptoms in patients with essential hypertension. However, the finding should be interpreted with caution because of the poor methodological quality of included trials. There is an urgent need for well-designed, long-term studies to assess the effectiveness of QJDHW in the treatment of essential hypertension.
\end{abstract}

\section{Introduction}

Hypertension is an increasingly prevalent chronic condition that is associated with serious morbidity and mortality. It is an important risk factor for the development and progression of cardiovascular disease, which is predicted that it will become the leading cause of death and disability worldwide by 2020 [1]. Hypertension is classified as either essential hypertension (EH) or secondary hypertension, and $\mathrm{EH}$ accounts for about 90-95\% of the cases characterized by high blood pressure with no obvious underlying medical causes [2]. In developing countries, it is a major medical concern that the high rate of undetected and untreated $\mathrm{EH}$ [3]. In China, the prevalence of $\mathrm{EH}$ is currently $18.8 \%$ [4]. The high rates of $\mathrm{EH}$, especially undiagnosed $\mathrm{EH}$, throughout the oriental countries double the risk of cardiovascular diseases, including coronary heart disease, congestive heart failure, ischemic and hemorrhagic stroke, renal failure, and peripheral arterial disease [5].

There is a growing tendency for people to turn to complementary and alternative medicine (CAM) $[6,7]$. In addition, the increasing prevalence of hypertension creates a broad market for alternative therapy to aid the management of blood pressure [8]. Several CAM clinical studies, including a substantial number of randomized controlled trials (RCTs) and systematic reviews, have shown that CAM is effective and safe for the treatment of hypertension [9-12]. It can also improve appetite, intestinal motility, metabolism, and emotional factors such as stress. Furthermore, studies showed 
that the application of CAM could enable tailored therapy in clinical practice, including lifestyle modification and individual choice of drugs lowering blood pressure $[13,14]$.

Qi Ju Di Huang Wan (QJDHW) decoction containing eight commonly used herbs (Chinese wolfberry fruit, chrysanthemum flower, common yam rhizome, tree peony bark, water plantain rhizome, cornus, and poria) had been used to relief symptoms like dizziness and vertigo for thousands of years in China. From the perspective of traditional Chinese medicine, it is believed that the mechanism of QJDHW may be related to calming the liver, suppressing liver yang hyperactivity, and nourishing kidney yin. Biochemically, QJDHW showed a good effect in decreasing the concentrations of angiotensin in plasma and myocardium, reducing the endothelin (ET) content and improving kidney blood stream in rats with essential hypertension [15-17].

Currently, it is common to see patients with essential hypertension seek QJDHW used alone or combined with antihypertensive agents as an alternative method. Recent studies also showed that QJDHW could help to control blood pressure $[18,19]$. However, the evidence examining the effectiveness of QJDHW for essential hypertension has never been systematically summarized. Thus, we performed this systematic review to critically assess the effectiveness of QJDHW for the treatment of essential hypertension.

\section{Methods}

2.1. Search Strategy. We searched the following sources up to January 2013: the Cochrane library, including the Cochrane Central Register of Controlled Trials (CENTRAL, 2012), PubMed, Chinese bases, including Chinese Biomedical Literature Database (CBM), Chinese National Knowledge Infrastructure (CNKI), and Chinese Scientific Journal Database (VIP). In addition, we also searched the databases of clinical trials such as Current Controlled Trials (http://www.controlled-trials.com/isrctn/), the National Centre for Complementary and Alternative Medicine (NCCAM) at the National Institutes of Health (NIH) (http://www.nccam.nih.gov/), and the Complementary and Alternative Medicine Specialist Library at the NHS National Library for Health (http://www.library.nhs.uk/cam/). The searching terms were "Qi Ju Di Huang Wan", "Yuan Fa Xing Gao Xue Ya (essential hypertension)", and "Gao Xue Ya (hypertension)". No language restriction was applied.

2.2. Inclusion Criteria and Exclusion Criteria. Our paper was restricted to RCTs that compared QJDHW or modified QJDHW, regardless of the preparation, with conventional antihypertensive drugs. RCTs comparing QJDHW combined antihypertensive drugs with antihypertensive drugs were also included. Quasi-RCTs were not considered. Animal studies, clinical trials including case report, case series traditional reviews were excluded. The main outcome measure was blood pressure (BP). The other outcome measures included the Scale for TCM Syndrome and Symptom Differentiation (TCM$\mathrm{SSD})$, the level of blood lipids (BL), plasma viscosity (PV), angiotensin II (AngII), endothelin (ET), calcitonin generelated peptide (CGRP), and safety. For TCM-SSD, the effect was presented as markedly effective, effective, and ineffective. Markedly effective was defined as the main symptoms such as headache, dizziness, palpitations, insomnia, tinnitus, and irritability disappeared or the TCM-SSD scores reduced rate $\geq 70 \%$; effective was defined as the main symptoms relieved or $70 \%>$ TCM-SSD scores reduced rate $\geq 30 \%$; ineffective was defined as the main symptoms that do not change or the TCM-SSD scores reduced rate $<30 \%$.

2.3. Study Selection and Data Extraction. The titles and abstracts of potentially relevant references were identified through the literature search and reviewed independently by 2 reviewers (G. Yang and Y. Zhang) according to predefined criteria. Discrepancies were resolved by consensus with another investigator (J. Wang). The following data were extracted: (1) citations (authors of study, year of publication), (2) methodological information, (3) participants information (sample size, age), (4) detailed information of interventions and controls, (5) outcome measures, and (6) adverse events.

2.4. Trial Quality Assessment. We assessed the methodological quality of included RCTs using Cochrane risk of bias tool. It has the following six domains: random sequence generation (selection bias), allocation concealment (selection bias), blinding of participants and personnel (performance bias), blinding of outcome data (attrition bias), incomplete outcome data (attrition bias), and selective reporting (reporting bias). The judgment was given as "high risk", "unclear risk", or "low risk": trials that met all the criteria were categorized as low risk of bias; those that met none of the criteria were categorized as high risk of bias; the others were categorized as an unclear risk of bias if insufficient information was available to make a judgment. Disagreements were resolved by discussion.

2.5. Data Analysis. The statistical package (RevMan 5.1.7) provided by Cochrane Collaboration was used for data analyses. Dichotomous data were expressed as risk ratio (RR) and continuous outcomes as weighted mean difference (WMD), with their 95\% confidence intervals (CI), respectively. Metaanalysis was performed if the intervention, control, and outcome were the same or similar. The statistical heterogeneity was examined with the $I^{2}$-test, where $I^{2}$ values of $50 \%$ or more were considered to be an indicator of a substantial heterogeneity. In the absence of significant heterogeneity, we pooled data using a fixed-effect model $\left(I^{2}<50 \%\right)$; otherwise we used random effects model $\left(I^{2}>50 \%\right)$ [20]. To maximize the similarities among studies that would be combined, and data were further stratified where possible into subgroups based on different types of interventions.

\section{Result}

3.1. Description of Included Trials. We identified 136 potentially relevant references from the electronic and manual searches. After screening the titles and abstracts, 94 studies 
were excluded because of duplicated publication (55 studies), animal studies (4 studies), and noncontrolled clinical trials (35 studies) including case report, case series, and traditional review. Full texts of 42 papers were retrieved, and finally 10 RCTs [21-30] were included. A total of 5 RCTs [21-25] were included in meta-analysis. All the RCTs were conducted in China and published in Chinese. The search for ongoing registered trials identified no trials (Figure 1).

The characteristics of the 10 RCTs are summarized in Table 1 . The total number of participants with essential hypertensive was 1024. The age of participants varied from 32 to 80 years. Five different diagnostic criteria of hypertension were used in the included trials: one trial [21] used 1999 WHOISH guidelines for the management of hypertension (1999 WHO-ISH GMH), one trial [23] used Seventh Report of the Joint National Committee on the Prevention, Detection, Evaluation, and Treatment of High Blood Pressure (JNC 7), one trial [22] used China Guidelines on Prevention and Management of High Blood Pressure-2005 (CGPMHBP2005), one trial [28] used CGPMHBP-2000, one trial [26] used 1978 WHO-ISH guidelines for the management of hypertension (1999 WHO-ISH GMH), and five trials [24, $25,27,29,30]$ only demonstrated patients with essential hypertension. Of the 10 trials, three trials $[21,23,26]$ reported TCM diagnostic criteria with yindeficiency and excessive yang syndrome and used Guidelines of Clinical Research of New Drugs of Traditional Chinese Medicine (GCRNDTCM); one trial [25] only demonstrated patients with yindeficiency and excessive yang syndrome in TCM, and six trials [2224, 27-30] have not reported TCM diagnostic criteria.

The interventions included Qi Ju Di Huang Wan in preparation of (modified) decoction and pill. The compositions of different QJDHW preparations are presented in Table 2. Of the 10 trials, 4 trials $[21,26,27,30]$ investigated the preparation of "Qi Ju Di Huang Wan" used alone versus antihypertensive drugs, and the rest 6 trials [22-25, 28, 29] compared the preparation of "Qi Ju Di Huang Wan" plus antihypertensive drugs versus antihypertensive drugs. The treatment duration ranged from 4 to 24 weeks.

3.2. Methodological Quality of Included Trials. The methodological quality of the 10 trials was generally low. All of the 10 included trials mentioned the randomized allocation of participants, and only two trials $[23,27]$ stated the methods of sequence generation of random number table. However, insufficient information was provided to judge whether it was conducted properly or not. Among the 10 trials, allocation concealment and double blind were not mentioned. None of the trials reported a dropout or withdrawae, and none of the trials reported sample size calculation. Since the protocols of all the 10 included trials were not accessible, selective reporting was generally unclear. In addition, no trial reported a followup (Figures 2 and 3).

\subsection{Effect of the Interventions}

3.3.1. “Qi Ju Di Huang Wan” versus Antihypertensive Drugs (Western Medicine). Four trials [21, 26, 27, 30] compared the preparation of "Qi Ju Di Huang Wan" used alone with antihypertensive drugs.

Blood Pressure. There was no significant difference between the two groups in systolic blood pressure (WMD: $0.10[-3.38$, 3.58]; $P=0.96$ ) and diastolic blood pressure (WMD: -0.20 $[-2.42,2.02] ; P=0.86$ ) after 8 weeks of treatment (see Tables 3 and 4).

TCM-SSD Scores. One trial [21] reported the TCM-SSD scores. The result showed a significant difference between QJDHW and antihypertensive drugs $(P=0.02)$.

3.3.2. "Qi Ju Di Huang Wan" Plus Antihypertensive Drugs versus Antihypertensive Drugs. Six trials [22-25, 28, 29] compared the combination of modified QJDHW plus antihyper tensive drugs with antihypertensive drugs.

Blood Pressure. Three trials [22-24] showed that there was a significant difference between treatment and control groups in systolic blood pressure (WMD: $-5.52[-8.96,-2.08] ; P=$ 0.002 ) and diastolic blood pressure (WMD: $-5.26[-6.83$, -3.70]; $P<0.00001)$. The forest plot was shown in the Figure 4 .

TCM-SSD Scores. Two trials [23, 25] reported the TCMSSD scores. The meta-analysis showed that the combination group had a beneficial effect on the improvement of TCM syndrome, compared to the antihypertensive drugs used alone (RR: $1.48[1.20,1.82] ; P=0$. 0.0003). We could not obtain more details of the TCM-SSD scores. So we just conducted an analysis of dichotomous data between groups (see Table 5).

3.3.3. Other Outcomes (BL, PV, AngII, ET, and CGRP). One trial [21] showed that after 8 weeks of treatment, the level of blood lipid (BL) and plasma viscosity (PV) decreased significantly $(P<0.05)$ in QJDHW group compared to captopril group. One trial [23] showed that after 4 weeks of treatment, the level of angiotensin II (Ang II), endothelin (ET) decreased significantly $(P<0.01)$ whereas the level of calcitonin gene-related peptide (CGRP) increased significantly $(P<0.05)$ in QJDH pill plus felodipine (plendil) group compared to felodipine (plendil) group. Furthermore, one trial [25] showed that after 8 weeks of treatment, the level of blood lipid (BL) decreased significantly $(P<0.05)$ in QJDH pill plus verapamil group compared to verapamil group.

\subsubsection{Sensitivity Analysis, Subgroup Analysis, and Publication} Bias. Due to no sufficient number of trials, we failed to conduct sensitivity analysis and subgroup analysis and also failed to perform funnel plot to detect publication bias.

3.4. Adverse Effect. Only one trial mentioned the adverse effect in captopril group such as dry cough [21]. Six trials [2126] reported no side effect in the QJDHW group compared to antihypertensive drugs. 


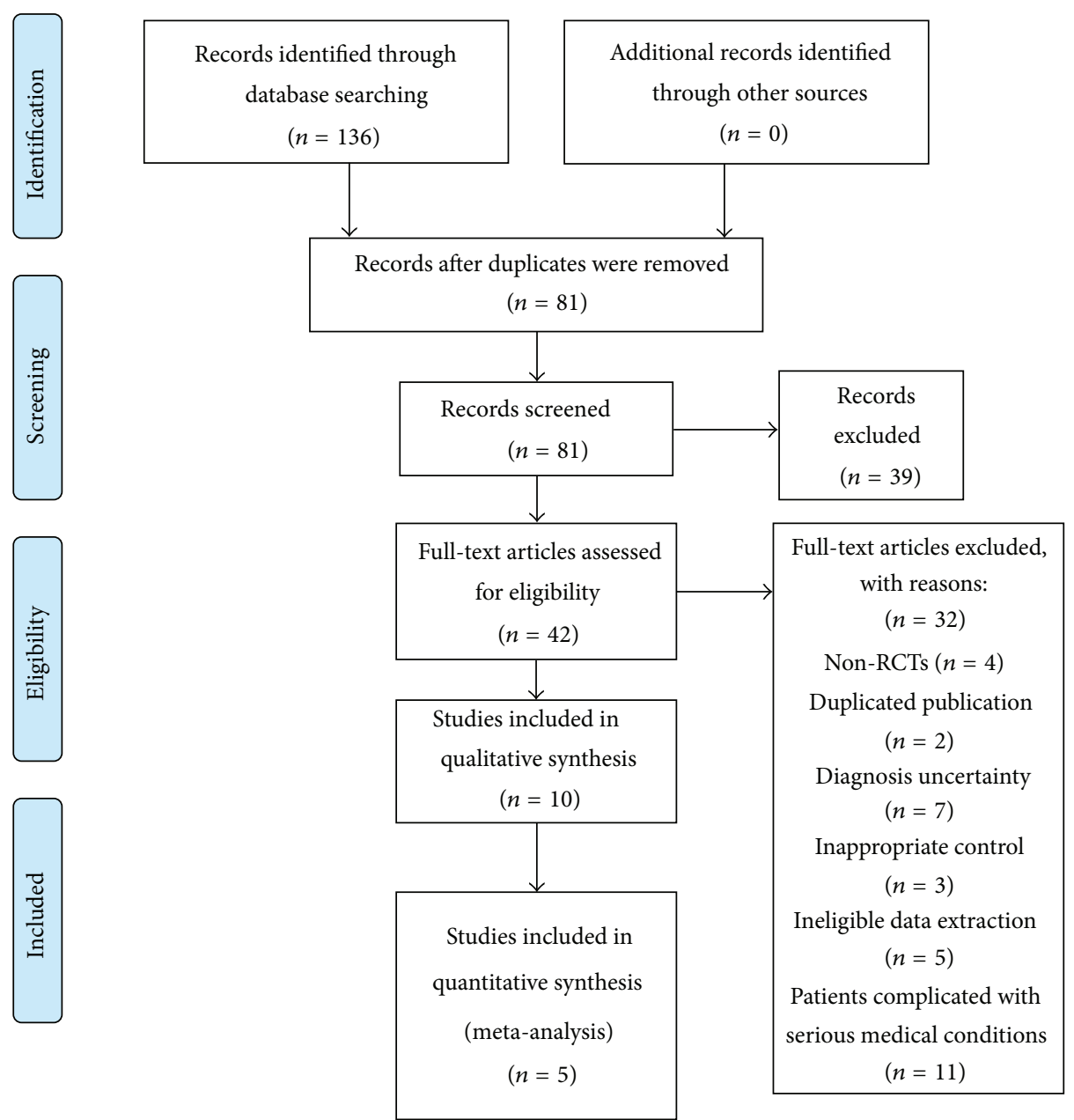

Figure 1: Flow Diagram of the literature searching and study selection.

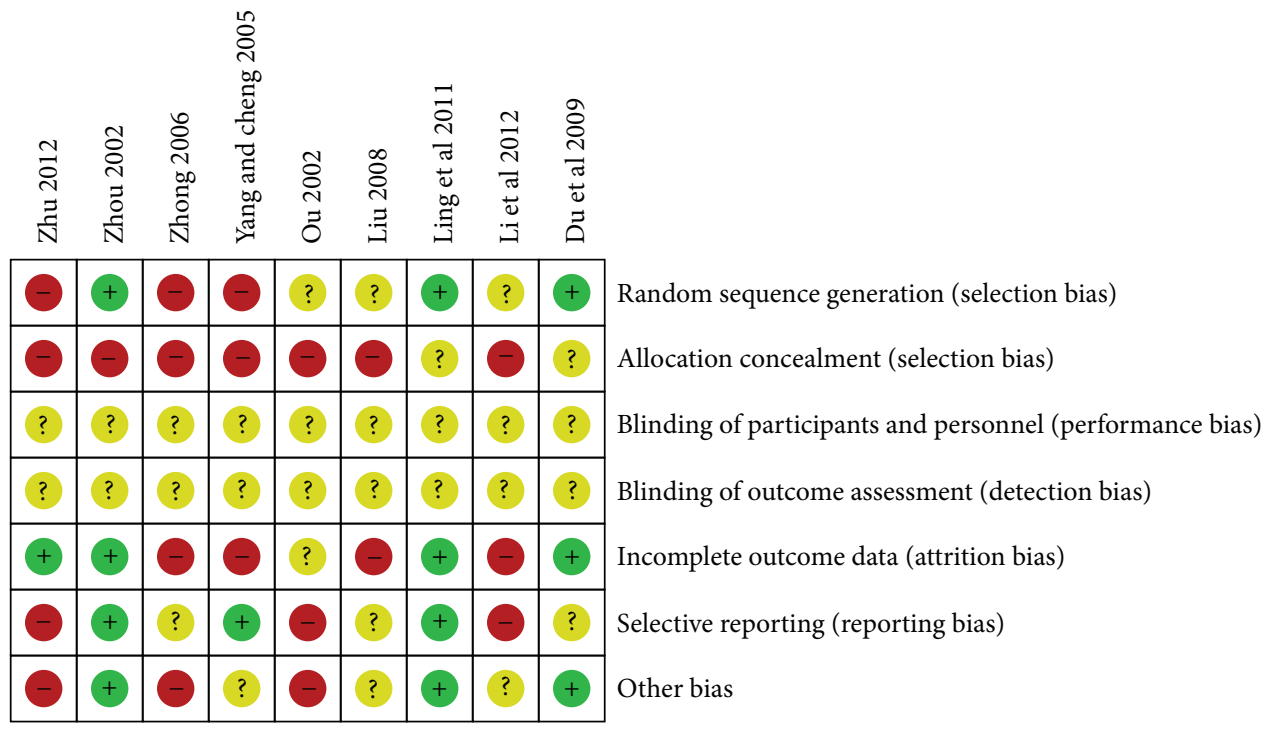

FIGURE 2: Risk of bias summary: review authors' judgments about each risk of bias item for each included study. 


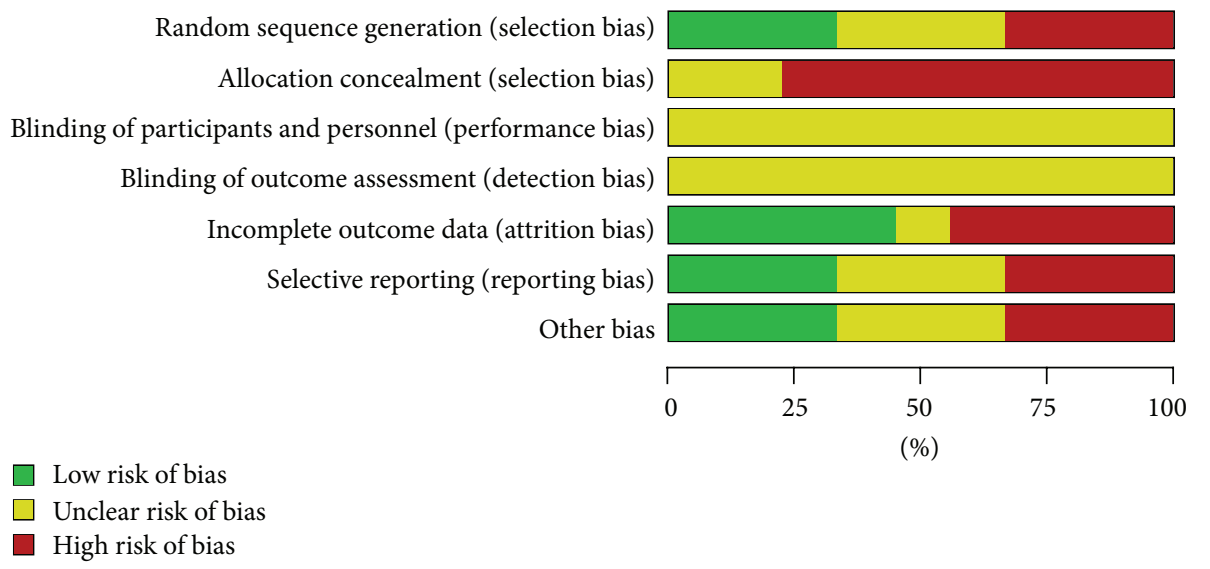

FIGURE 3: Risk of bias graph: review authors' judgments about each risk of bias item presented as percentages across all included studies.

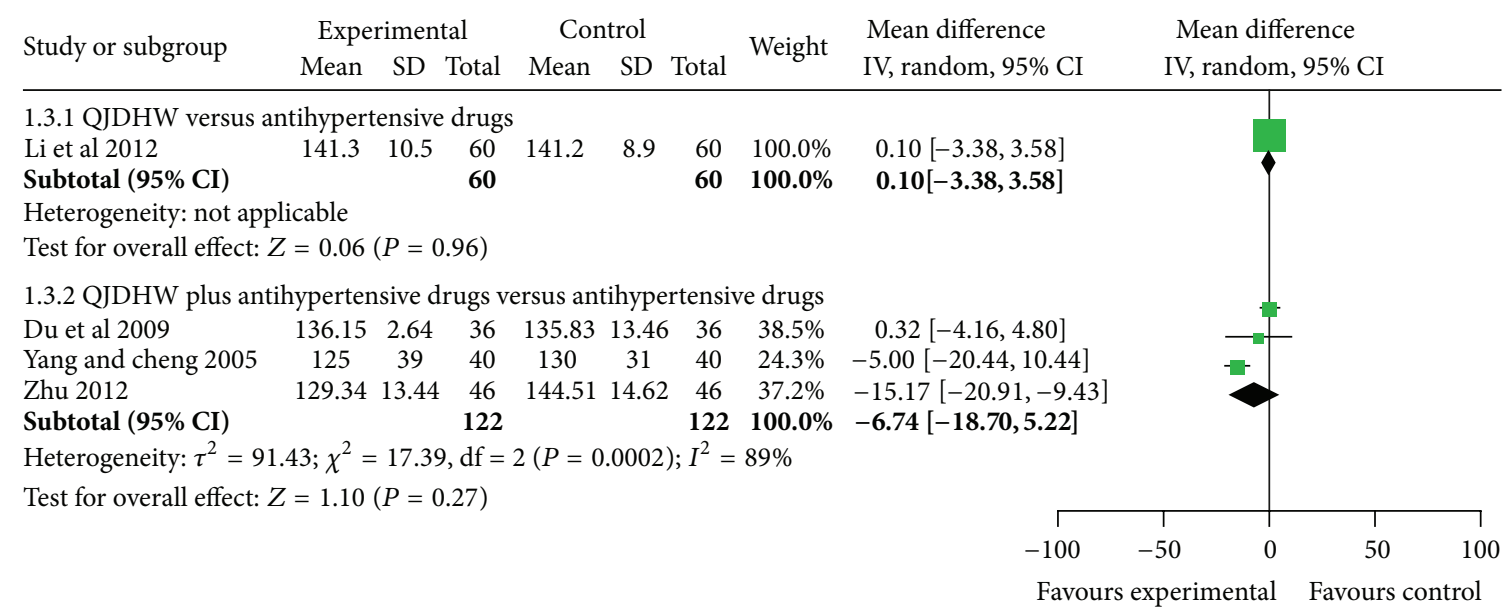

(a)

\begin{tabular}{|c|c|c|c|c|c|c|c|c|}
\hline \multirow{2}{*}{ Study or subgroup } & \multicolumn{2}{|c|}{ Experimental } & \multicolumn{3}{|c|}{ Control } & \multirow{2}{*}{ Weight } & \multirow{2}{*}{$\begin{array}{l}\text { Mean difference } \\
\text { IV, random, 95\% CI }\end{array}$} & \multirow{2}{*}{$\begin{array}{c}\text { Mean difference } \\
\text { IV, random, 95\% CI }\end{array}$} \\
\hline & Mean SD & Total & Mean & SD & Total & & & \\
\hline
\end{tabular}

1.4.1 QJDHW versus antihypertensive drugs

$\begin{array}{lllllllll}\text { Li et al } 2012 & 86.7 & 6.3 & 60 & 86.9 & 6.1 & 60 & 100.0 \% & -0.20[-2.42,2.02]\end{array}$

$\begin{array}{lllll}\text { Subtotal }(95 \% \mathrm{CI}) & 60 & 60 & 100.0 \% & -0.20[-2.42,2.02\end{array}$

Heterogeneity: not applicable

Test for overall effect: $Z=0.18(P=0.86)$

1.4.2 QJDHW plus antihypertensive drugs versus antihypertensive drugs

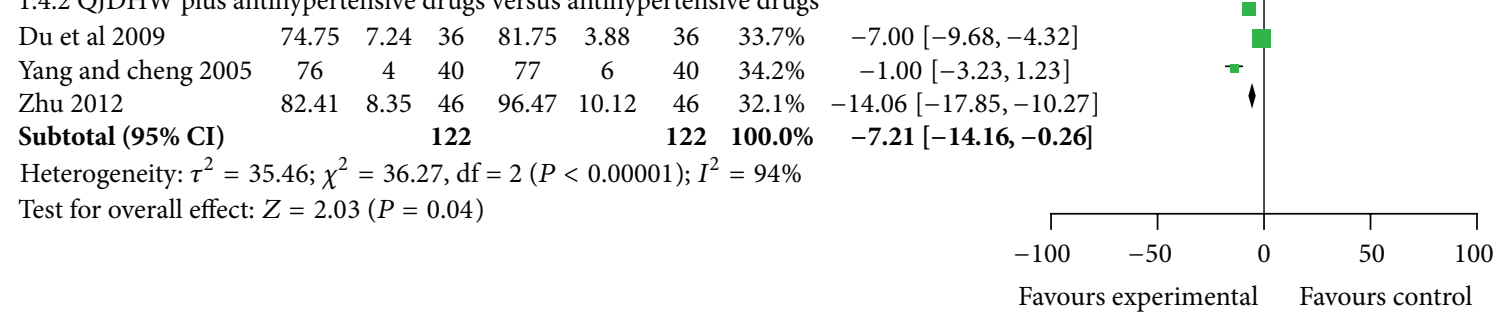

(b)

FIGURE 4: The forest plot of comparison of two groups for the outcome of blood pressure: (a) outcome of systolic blood pressure, (b) outcome of diastolic blood pressure. 
TABLE 1: Characteristics and methodological quality of included studies.

\begin{tabular}{|c|c|c|c|c|c|c|}
\hline Study ID & Sample & $\begin{array}{c}\text { Diagnosis } \\
\text { standard }\end{array}$ & Intervention & Control & $\begin{array}{l}\text { Course } \\
\text { (week) }\end{array}$ & Outcome measure \\
\hline Li et al., 2012 [21] & 120 & $\begin{array}{l}1999 \text { WHO-ISH GMH; } \\
\text { GCRNDTCM }\end{array}$ & QJDHW & Captopril & 8 & $\begin{array}{l}\text { BP; TCM-SSD; BL; PV; } \\
\text { side effect }\end{array}$ \\
\hline Zhu, 2012 [22] & 92 & CGPMHBP-2005 & $\begin{array}{l}\text { QJDH pill plus } \\
\text { nifedipine controlled } \\
\text { release tablet }\end{array}$ & $\begin{array}{c}\text { Nifedipine } \\
\text { controlled release } \\
\text { tablet }\end{array}$ & 8 & 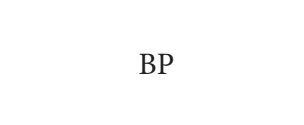 \\
\hline Du et al., 2009 [23] & 120 & JNC-7; GCRNDTCM & $\begin{array}{l}\text { QJDH pill plus } \\
\text { felodipine (plendil) }\end{array}$ & $\begin{array}{l}\text { Felodipine } \\
\text { (plendil) }\end{array}$ & 4 & $\begin{array}{l}\text { BP; TCM-SSD; } \\
\text { Ang II, ET, CGRP }\end{array}$ \\
\hline $\begin{array}{l}\text { Yang and Cheng, } \\
2005[24]\end{array}$ & 120 & $\begin{array}{l}\text { Hypertension diagnostic } \\
\text { criteria (unclear) }\end{array}$ & $\begin{array}{l}\text { modified QJDHW } \\
\text { plus metoprolol }\end{array}$ & Metoprolol & 8 & $\mathrm{BP}$ \\
\hline OuYang , 2002 [25] & 102 & $\begin{array}{c}\text { Hypertension diagnostic } \\
\text { criteria (unclear); TCM } \\
\text { diagnostic criteria (unclear) }\end{array}$ & $\begin{array}{l}\text { QJDH pill plus } \\
\text { verapamil }\end{array}$ & Verapamil & 8 & BP; TCM-SSD; BL \\
\hline Zhang, 1999 [26] & 70 & $\begin{array}{l}1999 \text { WHO-ISH GMH; } \\
\text { GCRNDTCM }\end{array}$ & QJDH pill & Nifedipine & 8 & $\mathrm{BP}$ \\
\hline Ling, 2011 [27] & 60 & $\begin{array}{l}\text { Hypertension diagnostic } \\
\text { criteria (unclear) }\end{array}$ & QJDH pill & Captopril & 4 & BP \\
\hline Liu, 2008 [28] & 180 & CGPMHBP-2000 & $\begin{array}{l}\text { QJDH pill plus } \\
\text { Western drugs }\end{array}$ & Western drugs & 24 & BP \\
\hline Zhong, 2006 [29] & 80 & $\begin{array}{l}\text { Hypertension diagnostic } \\
\text { criteria (unclear) }\end{array}$ & $\begin{array}{l}\text { QJDH pill plus } \\
\text { antihypertensive } \\
\text { tablets }\end{array}$ & $\begin{array}{l}\text { Antihypertensive } \\
\text { tablets }\end{array}$ & 4 & $\mathrm{BP}$ \\
\hline Zhou, 2002 [30] & 80 & $\begin{array}{l}\text { Hypertension diagnostic } \\
\text { criteria (unclear) }\end{array}$ & QJDH pill & $\begin{array}{c}\text { Nifedipine } \\
\text { controlled release } \\
\text { tablet }\end{array}$ & 4 & $\mathrm{BP}$ \\
\hline
\end{tabular}

Abbreviations: QJDHW: Qi Ju Di Huang Wan; WHO-ISH GMH: WHO-ISH guidelines for the management of hypertension; GCRNDTCM: Guidelines of Clinical Research of New Drugs of Traditional Chinese Medicine; CGPMHBP: China Guidelines on Prevention and Management of High Blood Pressure; JNC-7: Seventh Report of the Joint National Committee on the Prevention, Detection, Evaluation, and Treatment of High Blood Pressure; TCM: traditional Chinese medicine; TCM-SSD: TCM syndrome and symptom differentiation; BP: blood pressure; BL: blood lipid; PV: plasma viscosity; Ang II: angiotensin II; ET: endothelin; CGRP: calcitonin gene-related peptide.

\section{Discussion}

With the acceptance and popularity of CAM, the potential role of herbal remedies in global health care is being increasingly recognized in the recent years. Currently, more and more systematic reviews (SRs) and meta-analysis have been conducted to assess the efficiency of CAM for EH [3139]. It has made great contributions to the health and wellbeing of the people for the unique advantages of CAM in preventing and curing diseases, rehabilitation, and health care. To the best of our knowledge, this is the first systematic review and meta-analysis of RCTs for QJDHW in treating essential hypertension. Our review suggests that QJDHW may be effective for the treatment of hypertension. Based on the findings of meta-analyses of blood pressure and TCM-SSD scores, the preparation of "Qi Ju Di Huang Wan" including pill and decoction used alone or combined with antihypertensive drugs may have some beneficial effects on patients with essential hypertension. This review has the following limitations. Firstly, 5 databases have been searched up to January 2013 including the Cochrane library, PubMed, CBM, CNKI, and VIP. In addition, the databases of clinical trials such as Current Controlled Trials, the National Centre for Complementary and Alternative Medicine have also been searched without language restriction. However, all included trials were published in Chinese.

Secondly, the methodological quality of most included trials is generally low. Details of randomization were unclear. Concealment of allocation and blinding methods were not described, and reports of dropouts and withdrawals were incomplete. There were two trials (RCTs) [23, 27], stated randomization method through random number table. For the other five trials including Zhu 2012, Du et al. 2009, Yang and Cheng 2005, OuYang 2002, and Zhang 1999 [22-26], they only mentioned that "patients were randomized into two groups", with no detailed information of randomization generation. All of the included trials did not describe the blinding, we could not judge whether there were performance bias and detection bias because of the awareness of the therapeutic interventions for the subjective outcome measures [40-47]. All the included trials used blood pressure as a primary outcome measure, but half of the included trials evaluated the effectiveness with numerical values. The rest of the trials presented the effect as markedly effective, effective, and ineffective. We have tried to contact authors to get further information either by telephone or email. Unfortunately, no replies and information was got. We recommend that future researchers should follow the basic guidelines for 
TABLE 2: Composition of different QJDHW preparations.

\begin{tabular}{|c|c|c|}
\hline Study ID & Preparation & Composition \\
\hline Li et al., 2012 [21] & Decoction & $\begin{array}{l}\text { Chrysanthemum flower } 20 \mathrm{~g} \text {, Chinese wolfberry fruit } 15 \mathrm{~g} \text {, prepared } \\
\text { rehmannia root } 20 \mathrm{~g} \text {, Cornus } 15 \mathrm{~g} \text {, common yam rhizome } 15 \mathrm{~g} \text {, poria } \\
15 \mathrm{~g} \text {, water plantain rhizome } 12 \mathrm{~g} \text {, tree peony bark } 10 \mathrm{~g} \text {, danshen root } \\
15 \mathrm{~g} \text {, earth worm } 20 \mathrm{~g} \text {, red peony root } 12 \mathrm{~g} \text {, two-toothed achyranthes } \\
\text { root } 20 \mathrm{~g} \text {, gambir plant } 12 \mathrm{~g} \text {, and common self-heal fruit spike } 15 \mathrm{~g} \text {. } \\
\text { Severe dizziness and tinnitus plus dragon bones } 20 \mathrm{~g} \text { and oyster shell } \\
20 \mathrm{~g} \text {; vexing heat in the five centers and red tongue plus common } \\
\text { anemarrhena rhizome } 12 \mathrm{~g} \text { and Chinese wolfberry root bark } 12 \mathrm{~g} \text {; } \\
\text { amnesia and lumbago plus Tortoise plastron } 15 \mathrm{~g} \text {, Eucommia bark } \\
12 \mathrm{~g} \text {, Chinese taxillus herb } 15 \mathrm{~g} \text {, and deer antler glue } 10 \mathrm{~g} \text { (melted in } \\
\text { decoction). }\end{array}$ \\
\hline Zhu, 2012 [22] & pill & Chinese patent medicine \\
\hline Du et al., 2009 [23] & pill & Chinese patent medicine \\
\hline $\begin{array}{l}\text { Yang and Cheng, } 2005 \\
\text { [24] }\end{array}$ & Modified QJDHW & $\begin{array}{l}\text { Chrysanthemum flower } 20 \mathrm{~g} \text {, Chinese wolfberry fruit } 15 \mathrm{~g} \text {, prepared } \\
\text { rehmannia root } 20 \mathrm{~g} \text {, Cornus } 15 \mathrm{~g} \text {, common yam rhizome } 15 \mathrm{~g} \text {, poria } \\
15 \mathrm{~g} \text {, water plantain rhizome } 12 \mathrm{~g} \text {, and tree peony bark } 10 \mathrm{~g} \text {. }\end{array}$ \\
\hline OuYang, 2002 [25] & pill & Chinese patent medicine \\
\hline Zhang, 1999 [26] & pill & Chinese patent medicine \\
\hline Ling, $2011[27]$ & pill & Chinese patent medicine \\
\hline Liu, 2008 [28] & pill & Chinese patent medicine \\
\hline Zhong, 2006 [29] & pill & Chinese patent medicine \\
\hline Zhou, $2002[30]$ & pill & Chinese patent medicine \\
\hline
\end{tabular}

Abbreviations: QJDHW: Qi Ju Di Huang Wan.

TABLE 3: Analyses of systolic blood pressure.

\begin{tabular}{|c|c|c|c|}
\hline Trials & & WMD $[95 \% \mathrm{CI}]$ & $P$ value \\
\hline \multicolumn{4}{|l|}{ QJDHW versus antihypertensive drugs } \\
\hline QJDHW versus captopril & 1 & $0.10[-3.38,3.58]$ & 0.96 \\
\hline Meta-Analysis & 1 & $0.10[-3.38,3.58]$ & 0.96 \\
\hline \multicolumn{4}{|l|}{ QJDHW plus antihypertensive drugs versus antihypertensive drugs } \\
\hline QJDH pill plus nifedipine controlled release tablet versus nifedipine controlled release tablet & 1 & $-15.17[-20.91,-9.43]$ & $<0.00001$ \\
\hline QJDH pill plus felodipine (plendil) versus felodipine (plendil) & 1 & $0.32[-4.16,4.80]$ & 0.89 \\
\hline Modified QJDHW plus metoprolol versus metoprolol & 1 & $-5.00[-20.44,10.44]$ & 0.53 \\
\hline Meta-Analysis & 3 & $-5.52[-8.96,-2.08]$ & 0.002 \\
\hline
\end{tabular}

Abbreviations: QJDHW: Qi Ju Di Huang Wan.

TABLE 4: Analyses of diastolic blood pressure.

\begin{tabular}{|c|c|c|c|}
\hline Trials & & WMD $[95 \% \mathrm{CI}]$ & $P$ value \\
\hline \multicolumn{4}{|l|}{ QJDHW versus antihypertensive drugs } \\
\hline QJDHW versus captopril & 1 & $-0.20[-2.42,2.02]$ & 0.86 \\
\hline Meta-Analysis & 1 & $-0.20[-2.42,2.02]$ & 0.86 \\
\hline \multicolumn{4}{|l|}{ QJDHW plus antihypertensive drugs versus antihypertensive drugs } \\
\hline QJDH pill plus nifedipine controlled release tablet versus nifedipine controlled release tablet & 1 & $-14.06[-17.85,-10.27]$ & $<0.00001$ \\
\hline QJDH pill plus felodipine (plendil) versus felodipine (plendil) & 1 & $-7.00[-9.68,-4.32]$ & $<0.00001$ \\
\hline Modified QJDHW plus metoprolol versus metoprolol & 1 & $-1.00[-3.23,1.23]$ & 0.38 \\
\hline Meta-Analysis & 3 & $-5.26[-6.83,-3.70]$ & $<0.00001$ \\
\hline
\end{tabular}

Abbreviations: QJDHW: Qi Ju Di Huang Wan. 
TABLE 5: Analyses of TCM-SSD Scores.

\begin{tabular}{|c|c|c|c|c|c|}
\hline Trials & & Intervention $(n / N)$ & Control $(n / N)$ & $\mathrm{RR}[95 \% \mathrm{CI}]$ & $P$ value \\
\hline \multicolumn{6}{|l|}{ QJDHW versus antihypertensive drugs } \\
\hline QJDHW versus captopril & 1 & $56 / 60$ & $47 / 60$ & $1.19[1.03,1.38]$ & 0.02 \\
\hline Meta-Analysis & 1 & $56 / 60$ & $47 / 60$ & $1.19[1.03,1.38]$ & 0.02 \\
\hline \multicolumn{6}{|l|}{ QJDHW plus antihypertensive drugs versus antihypertensive drugs } \\
\hline QJDH pill plus felodipine (plendil) versus felodipine (plendil) & 1 & $31 / 36$ & $24 / 36$ & $1.29[0.99,1.8]$ & 0.06 \\
\hline QJDH pill plus verapamil versus verapamil & 1 & $31 / 34$ & $18 / 34$ & $1.72[1.23,2.40]$ & 0.001 \\
\hline Meta-Analysis & 2 & $62 / 70$ & $42 / 70$ & $1.48[1.20,1.82]$ & 0.0003 \\
\hline
\end{tabular}

Abbreviations: QJDHW: Qi Ju Di Huang Wan.

reporting clinical trials such as the Consolidated Standards of Reporting Trials (CONSORT) statement.

Thirdly, the treatment duration of most included studies was short, varying from 4 to 8 weeks. Only one trial has a long duration of 24 weeks. Since hypertension is a chronic condition, it is a great concern of patients about the effect of long-term treatment. Indeed, none of the included trials reported the mortality rate or the incidence of complications. Moreover, hypertension may exacerbate with or without treatment, especially in China; a major risk factor for hypertension is unbalanced intake of dietary sodium and potassium. Studies have indicated that high dietary sodium intake may change the circadian rhythm of 24-hour blood pressure, which is characterized by a higher nighttime blood pressure. The prevalence of isolated nighttime hypertension, which is defined as a nighttime systolic/diastolic blood pressure more than $120 / 70 \mathrm{~mm} \mathrm{Hg}$ and a daytime systolic/diastolic blood pressure less than $135 / 85 \mathrm{~mm} \mathrm{Hg}$, is higher in Chinese than in Europeans $[48,49]$. Therefore, a longer follow-up period with serial measurements of outcomes is suggested to determine the long-term effectiveness of QIDHW prescriptions. Thus, RCTs of QJDHW prescriptions with design to measure the followup of outcomes are urgently needed.

Fourthly, among the included trials, there is inadequate reporting on adverse events. None of the five trials reported information on the adverse effect of QJDHW decoctions or pills. Due to the limited information of adverse events, we could not draw definite conclusions on the safety of QJDHW prescriptions. Though most of Chinese herbal medicines and Chinese patent medicines are widely accepted and safely used, the increasing reports of liver and kidney toxicity and other adverse events related to Chinese medicines [50-59] draw much attention to the concern of safety. We recommend that future clinical trials should use QJDHW prescriptions with caution and report adverse events appropriately.

Fifthly, there may be publication bias in this review. We doubted whether all the RCTs have positive effect of QJDHW prescriptions when analyzed by standard statistical techniques using risk ratios or mean differences. For this, extensive searches for unpublished material have been conducted, but no unpublished "negative" studies were found.

In general, comparing to three categories (calcium antagonist, beta blocker, and angiotensin-converting enzyme inhibitors) of antihypertensive drugs such as captopril, nifedipine, felodipine (plendil), metoprolol, and verapamil, the preparation of QJDHW appears to lower blood pressure and improve the symptoms with less adverse event. The combination of QJDHW and antihypertensive drugs may have significant effectiveness compared to antihypertensive drugs used alone. However, the quality of RCTs included in our review was limited for us to draw definite conclusions about QJDHW. More rigorous RCTs are required to be performed to prove the effectiveness of QJDHW for treating hypertension.

\section{Conclusions}

Our review indicates that QJDHW for treating essential hypertension has some beneficial effects compared to antihypertensive drugs, although the results are of limited value due to the clinical heterogeneity and low methodological quality of the included studies which prevent us from drawing a definitive conclusion for the effectiveness of QJDHW. However, QJDHW preparations are relatively safe, as a traditional Chinese medical therapy for improving symptoms of essential hypertension. Nevertheless, questions that cannot be conclusively answered at present include whether QIDHW prescriptions should be widely recommended and what the most effective preparation of QJDHW is. More well-designed, long-term clinical trials are needed.

\section{Conflict of Interests}

All authors declare that they have no conflict of interests.

\section{Authors' Contribution}

Xiaochen Yang, Xingjiang Xiong, and Guoyan Yang contributed equally to this paper.

\section{Acknowledgments}

The current work was partially supported by the National Basic Research Program of China (973 Program, no. 2003CB517103) and the National Natural Science Foundation Project of China (no. 90209011). The funders had no role in the study design, data collection and analysis, decision to publish, or preparation of the paper. 


\section{References}

[1] World Health Organization, 2008-2013 action plan for the global strategy for the prevention and control of noncommunicable diseases, 2008, http://www.who.int/nmh/Actionplan-PCNCD-2008.pdf.

[2] O. A. Carretero and S. Oparil, "Essential hypertension. Part I: definition and etiology," Circulation, vol. 101, no. 3, pp. 329-335, 2000.

[3] D. O. Abegunde, C. D. Mathers, T. Adam, M. Ortegon, and K. Strong, "The burden and costs of chronic diseases in lowincome and middle-income countries," Lancet, vol. 370, no. 9603, pp. 1929-1938, 2007.

[4] J. G. Wang and Y. Li, "Characteristics of hypertension in the Chinese population," Current Hypertension Reports, vol. 14, no. 5, pp. 410-415, 2012.

[5] S. Stewart and K. Sliwa, "Preventing CVD in resource-poor areas: perspectives from the 'real-world"' Nature Reviews Cardiology, vol. 6, pp. 489-492, 2009.

[6] E. Ernst, "Prevalence of use of complementary/alternative medicine: a systematic review," Bulletin of the World Health Organization, vol. 78, no. 2, pp. 252-257, 2000.

[7] K. J. Chen, K. K. Hui, M. S. Lee, and H. Xu, “The potential benefit of complementary/alternative medicine in cardiovascular diseases," Evidence-Based Complementary and Alternative Medicine, vol. 2012, Article ID 125029, 1 page, 2012.

[8] E. Ernst, K. L. Resch, S. Mills et al., "Complementary medicine-a definition," British Journal of General Practice, vol. 45, pp. 445-506, 1995.

[9] M. S. Lee, T. Y. Choi, B. C. Shin et al., "Cupping for hypertension: a systematic review," Clinical and Experimental Hypertension, vol. 32, no. 7, pp. 423-425, 2010.

[10] M. H. Hur, M. S. Lee, C. Kim, and E. Ernst, "Aromatherapy for treatment of hypertension: a systematic review," Journal of Evaluation in Clinical Practice, vol. 18, no. 1, pp. 37-41, 2012.

[11] J. I. Kim, J. Y. Choi, H. Lee et al., "Moxibustion for hypertension: a systematic review," BMC Cardiovasc Disord, vol. 10, article 33, 2010.

[12] M. S. Lee, E. N. Lee, J. I. Kim, and E. Ernst, "Tai chi for lowering resting blood pressure in the elderly: a systematic review," Journal of Evaluation in Clinical Practice, vol. 16, no. 4, pp. 818-824, 2010.

[13] J. Wang, P. Q. Wang, and X. J. Xiong, "Current situation and reunderstanding of syndrome and formula syndrome in Chinese medicine," Internal Medicine, vol. 2, no. 3, article 113, 2012.

[14] H. Xu and K. Chen, "Integrative medicine: the experience from China," Journal of Alternative and Complementary Medicine, vol. 14, no. 1, pp. 3-7, 2008.

[15] Z. J. Yue, Z. D. Zou, D. H. Li et al., "Effect of reinforcing kidney on blood pressure and kidney blood stream in SHR," Zhong Guo Shi Yan Fang Ji Xue Za Zhi, vol. 15, no. 1, pp. 42-44, 2009.

[16] F. F. Duo, Z. D. Zou, W. J. Wang et al., "Effect of Qi Ju Di Huang Wan on injured vascular endothelial cell ultrastructure induced by Ang II," Zhong Guo Yi Yao Dao Kan, vol. 10, 2010.

[17] Z. J. Yue, Z. D. Zou, D. H. Li et al., "Effect of reinforcing kidney on blood pressure and kidney function in SHR," Zhong Guo Shi Yan Fang Ji Xue Za Zhi, vol. 15, no. 8, pp. 63-65, 2009.

[18] J. Wang and X. J. Xiong, "Outcome measures of Chinese herbal medicine for hypertension: an overview of systematic reviews," Evidence-Based Complementary and Alternative Medicine, vol. 2012, Article ID 697237, 7 pages, 2012.
[19] X. J. Xiong, X. C. Yang, Y. M. Liu et al., "Chinese herbal formulas for treating hypertension in traditional Chinese medicine: perspective of modern science," Hypertension Research, 2013.

[20] J. P. T. Higgins and S. Green, Corchrane Reviewers' Handbook 5.1.0, Version 5.1.0, Review Manager (RevMan) [Computer program], 2011.

[21] W. Li, H. G. Chen, and W. You, "Clinical effect of Qi Ju Di Huang decoction on 60 patients with essential hypertension," Zhong Guo Min Jian Liao Fa Za Zhi, vol. 20, no. 2, pp. 37-38, 2012.

[22] C. Q. Zhu, “The effect of Qi Ju Di Huang Wan on elderly essential hypertension combined with nifedipine controlled release tablet," Yao Wu Yu Lin Chuang Za Zhi, vol. 2, no. 2, pp. 119-121, 2012.

[23] B. J. Du, Z. F. Huang, and X. C. Kong, "Effects of Qi Ju Di Huang Wan combined with felodipine on angiotensin, endothelin and CGRP on patients with essential hypertension," Zhong Yi Yao Xin Xi Za Zhi, vol. 26, no. 4, pp. 53-54, 2009.

[24] S. Yang and Y. P. Cheng, "The effect of Qi Ju Di Huang Wan on essential hypertension combined with metoprolol," Lin Chuang Hui Cui Za Zhi, vol. 20, no. 17, pp. 1005-1006, 2005.

[25] Y. P. OuYang, "Clinical effect of Qi Ju Di Huang Wan combined with verapamil on 34 patients with elderly essential hypertension," Hu Nan Zhong Yi Yao Dao Bao, vol. 8, no. 1, pp. 15-16, 2002.

[26] Q. Z. Zhang, "Clinical effect of Qi Ju Di Huang Wan on patients with decifiecy of yin of essential hypertension," Shaanxi Zhong Yi Yao, vol. 7, pp. 987-990, 1999.

[27] C. D. Ling, "Clinical effect of Qi Ju Di Huang Wan combined with Ni Fuda on 30 patients with essential hypertension," Shi Yong Zhong Yi Yao, vol. 7, pp. 325-329, 2011.

[28] Y. L. Liu, “The impact of Chinese medicine against male sexual dysfunction due to hypertension," He Nan Zhong Yi Yao Xue Bao, vol. 16, pp. 1256-1259, 2008.

[29] Y. Zhong, "Clinical effect of Qi Ju Di Huang Wan on 50 patients with essential hypertension," Zhong Yi Za Zhi, vol. 17, pp. 856860, 2006.

[30] Y. F. Zhou, "Clinical effect of Qi Ju Di Huang Wan on 80 patients with decifiecy of yin of essential hypertension," Shaanxi Zhong Yi Yao, vol. 8, pp. 532-539, 2002.

[31] J. Wang, B. Feng, X. C. Yang et al., "Tianma gouteng yin as adjunctive treatment for essential hypertension: a systematic review of randomized controlled trials," Evidence-Based Complementary and Alternative Medicine, vol. 2013, Article ID 706125, 18 pages, 2013.

[32] X. J. Xiong, X. C. Yang, B. Feng et al., "Zhen gan xi feng decoction, a traditional Chinese herbal formula, for the treatment of essential hypertension: a systematic review of randomized controlled trials," Evidence-Based Complementary and Alternative Medicine, vol. 2013, Article ID 982380, 9 pages, 2013.

[33] J. Wang, X. C. Yang, B. Feng et al., "Is Yangxue Qingnao Granule combined with antihypertensive drugs, a new integrative medicine therapy, more effective than antihypertensive therapy alone in treating essential hypertension?" EvidenceBased Complementary and Alternative Medicine, vol. 2013, Article ID 540613, 8 pages, 2013.

[34] X. J. Xiong, X. C. Yang, W. Liu et al., "Banxia baizhu tianma decoction for essential hypertension: a systematic review of randomized controlled trials," Evidence-Based Complementary and Alternative Medicine, vol. 2012, Article ID 271462, 10 pages, 2012. 
[35] J. Wang, K. W. Yao, X. C. Yang et al., "Chinese patent medicine liu wei di huang wan combined with antihypertensive drugs, a new integrative medicine therapy, for the treatment of essential hypertension: a systematic review of randomized controlled trials," Evidence-Based Complementary and Alternative Medicine, vol. 2012, Article ID 714805, 7 pages, 2012.

[36] J. Wang and X. J. Xiong, "Current situation and perspectives of clinical study in integrative medicine in China," Evidence-Based Complementary and Alternative Medicine, vol. 2012, Article ID 268542, 11 pages, 2012.

[37] A. Ferreira, "Integrative medicine for hypertension: the earlier the better for treating who and what are not yet ill," Hypertension Research, 2013.

[38] M. S. Lee, H. J. Lim, M. S. Lee, and H. S. Jang, "Perceptions, knowledge and misuse of an oriental herbal drug: a survey of 608 Korean female nursing college students," Complementary Therapies in Clinical Practice, vol. 11, no. 3, pp. 200-204, 2005.

[39] M. S. Ju, S. Lee, I. Bae et al., "Effects of aroma massage on home blood pressure, ambulatory blood pressure, and sleep quality in middle-aged women with hypertension," Evidence-Based Complementary and Alternative Medicine, vol. 2013, Article ID 403251, 7 pages, 2013.

[40] K. F. Schulz, L. Chalmers, R. J. Hayes, and D. G. Altman, "Empirical evidence of bias: dimensions of methodological quality associated with estimates of treatment effects in controlled trials," Journal of the American Medical Association, vol. 273, no. 5, pp. 408-412, 1995.

[41] J. Hu, J. Zhang, W. Zhao, Y. Zhang, L. Zhang, and H. Shang, "Cochrane systematic reviews of Chinese herbal medicines: an overview," PLoS ONE, vol. 6, no. 12, Article ID e28696, 2011.

[42] L. Liu, "The clinical trial barriers," Nature, vol. 480, no. 7378, article S100, 2011.

[43] Y. Tu, "The discovery of artemisinin (qinghaosu) and gifts from Chinese medicine," Nature Medicine, vol. 17, no. 10, pp. 12171220, 2011.

[44] W. Chen, C. E. D. Lim, H. J. Kang, and J. Liu, "Chinese herbal medicines for the treatment of type A H1N1 influenza: a systematic review of randomized controlled trials," PLOS ONE, vol. 6, no. 12, Article ID e28093, 2011.

[45] M. Y. Liu and K. J. Chen, "Convergence: the tradition and the modern," Chinese Journal of Integrative Medicine, vol. 18, no. 3, pp. 164-165, 2012.

[46] Z. Junhua, S. Hongcai, G. Xiumei et al., "Methodology and reporting quality of systematic review/meta-analysis of traditional Chinese medicine," Journal of Alternative and Complementary Medicine, vol. 13, no. 8, pp. 797-805, 2007.

[47] J. Wang and X. J. Xiong, "Control strategy on hypertension in Chinese medicine," Evidence-Based Complementary and Alternative Medicine, vol. 2012, Article ID 284847, 6 pages, 2012.

[48] R. C. Hermida, M. H. Smolensky, D. E. Ayala et al., "2013 ambulatory blood pressure monitoring recommendations for the diagnosis of adult hypertension, assessment of cardiovascular and other hypertension-associated risk, and attainment of therapeutic goals," Chronobiology International, vol. 30, no. 3, pp. 355-410, 2013.

[49] B. Wizner, D. G. Dechering, L. Thijs et al., "Short-term and long-term repeatability of the morning blood pressure in older patients with isolated systolic hypertension," Journal of Hypertension, vol. 26, no. 7, pp. 1328-1335, 2008.

[50] X. J. Xiong, F. Y. Chu, H. X. Li, and Q. Y. He, "Clinical application of the TCM classic formulae for treating chronic bronchitis,"
Journal of Traditional Chinese Medicine, vol. 31, no. 1, pp. 69-72, 2011.

[51] J. Wang, R. van der Heijden, S. Spruit et al., "Quality and safety of Chinese herbal medicines guided by a systems biology perspective," Journal of Ethnopharmacology, vol. 126, no. 1, pp. 31-41, 2009.

[52] K. Chan, "Some aspects of toxic contaminants in herbal medicines," Chemosphere, vol. 52, no. 9, pp. 1361-1371, 2003.

[53] D. Melchart, K. Linde, S. Hager, D. Shaw, and R. Bauer, "Liver enzyme elevations in patients treated with traditional Chinese medicine," Journal of the American Medical Association, vol. 282, no. 1, pp. 28-29, 1999.

[54] L. G. Miller, "Herbal medicinals: selected clinical considerations focusing on known or potential drug-herb interactions," Archives of Internal Medicine, vol. 158, no. 20, pp. 2200-2211, 1998.

[55] A. Tachjian, V. Maria, and A. Jahangir, "Use of herbal products and potential interactions in patients with cardiovascular diseases," Journal of the American College of Cardiology, vol. 55, no. 6, pp. 515-525, 2010.

[56] Z. Y. Shen and X. Chen, "Analysis on 99 cases of adverse reactions of Chinese patent drugs," African Journal of Microbiology Research, vol. 6, no. 8, pp. 1742-1746, 2012.

[57] H. Xu and K. J. Chen, "Herb-drug interaction: an emerging issue of integrative medicine," Chinese Journal of Integrative Medicine, vol. 16, no. 3, pp. 195-196, 2010.

[58] P. Windrum, D. R. Hull, and T. C. M. Morris, "Herb-drug interactions," Lancet, vol. 355, no. 9208, pp. 1019-1020, 2000.

[59] A. Fugh-Berman, "Herb-drug interactions," Lancet, vol. 355, no. 9198, pp. 134-138, 2000. 


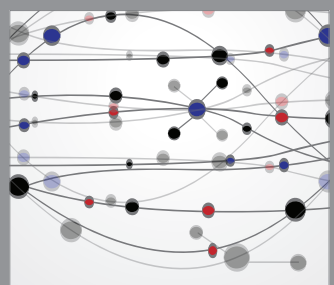

The Scientific World Journal
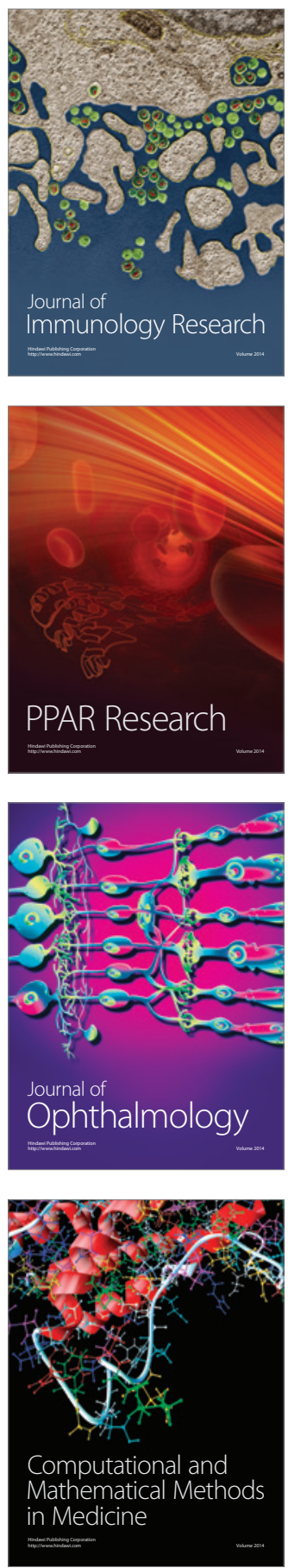

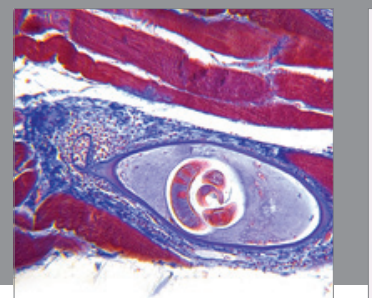

Gastroenterology

Research and Practice
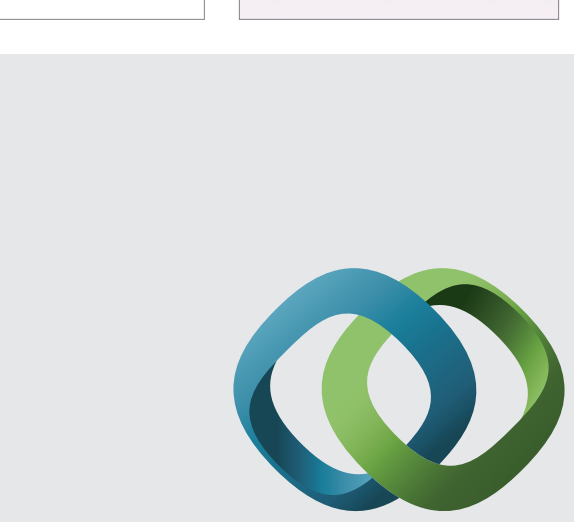

\section{Hindawi}

Submit your manuscripts at

http://www.hindawi.com
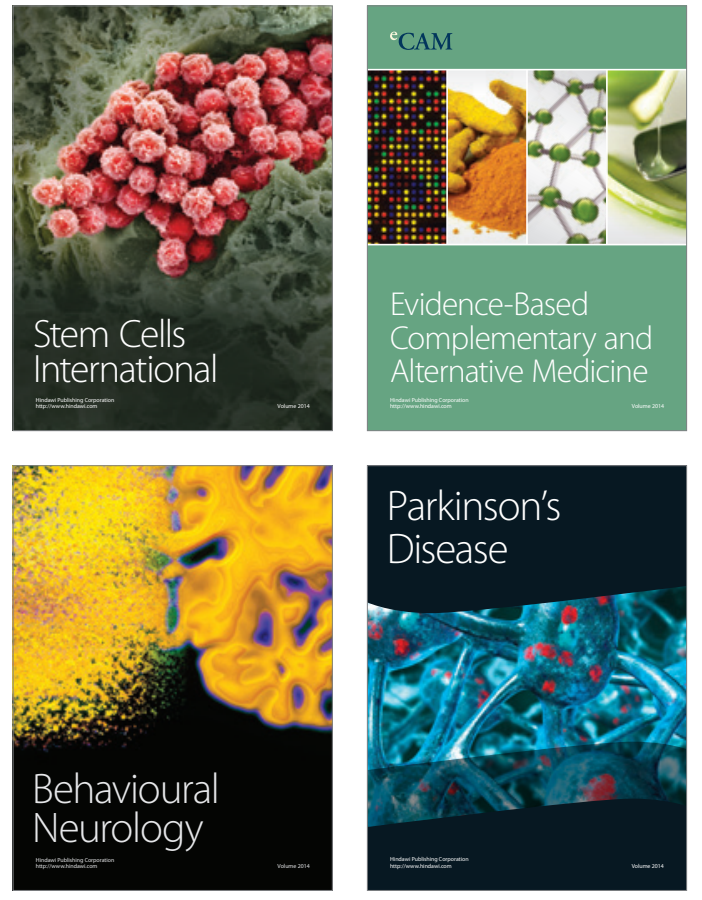
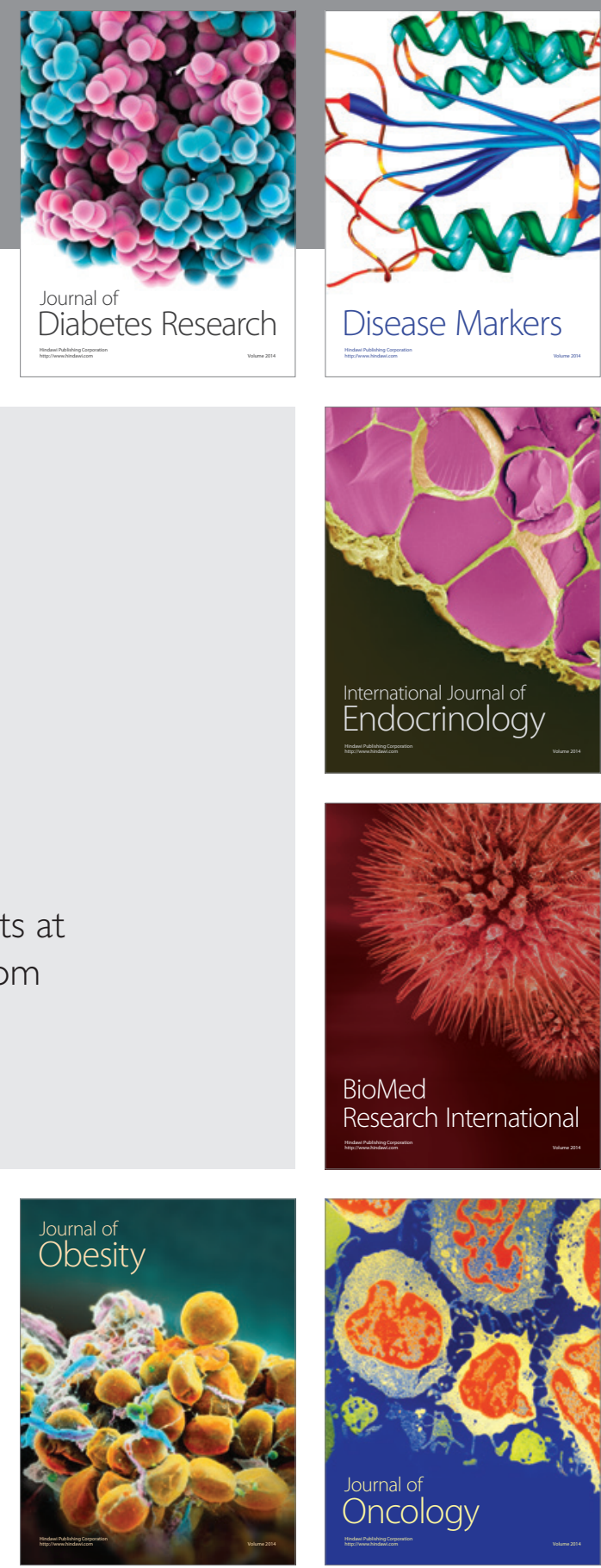

Disease Markers
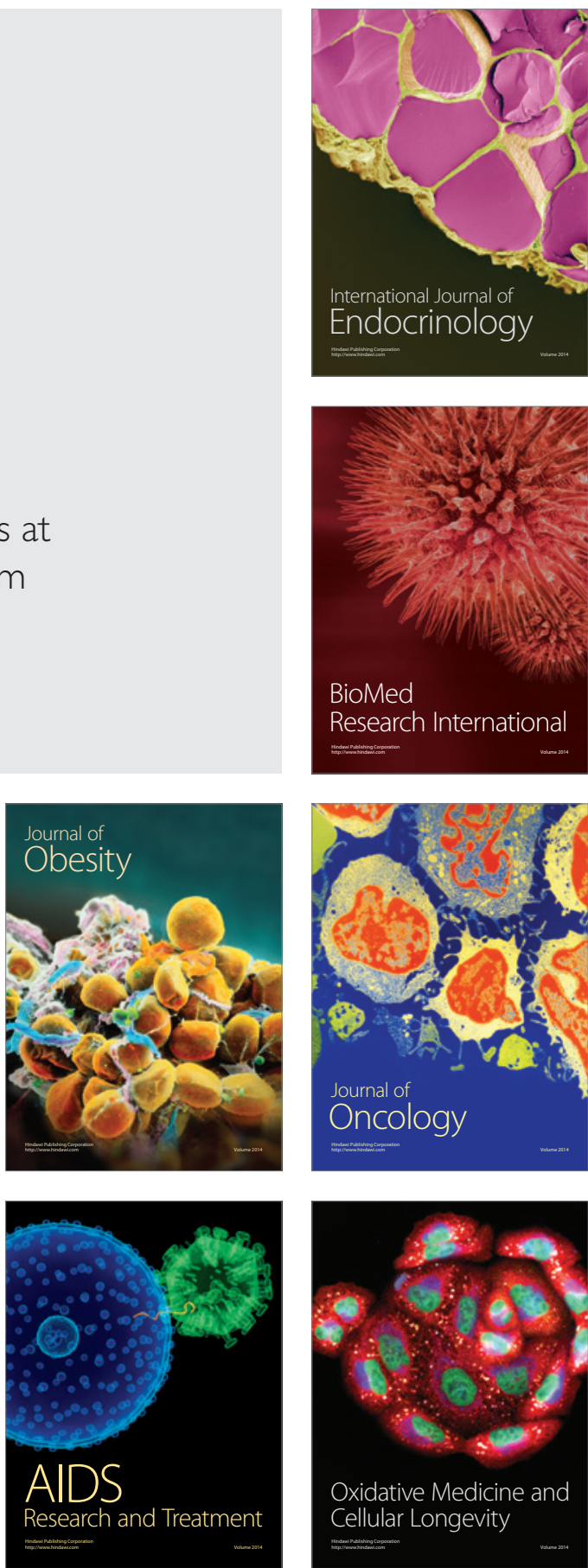